\title{
Validity of a Screening Question for Head Tremor: An Analysis of Four Essential Tremor Case Samples
}

\author{
Elan D. Louis ${ }^{\text {a-d }}$ Monika Michalec ${ }^{\text {a }}$ \\ ${ }^{\mathrm{a}} \mathrm{GH}$ Sergievsky Center, ${ }^{\mathrm{b}}$ Department of Neurology, College of Physicians and Surgeons, ${ }^{\mathrm{C}}$ Department of \\ Epidemiology, Mailman School of Public Health, and ${ }^{\mathrm{d}}$ Taub Institute for Research on Alzheimer's Disease and \\ the Aging Brain, College of Physicians and Surgeons, Columbia University, New York, N.Y., USA
}

\section{Key Words}

Essential tremor · Epidemiology · Clinical · Screening ·

Validity $\cdot$ Head tremor

\section{Abstract}

Background: The goal of this study was to assess the validity of a screening question for head tremor in essential tremor (ET). There are no published data on this topic, and the knowledge will guide future epidemiological investigations of this disorder. Methods: These analyses utilized four distinct patient samples: a population-based study in northern Manhattan, a study of the environmental epidemiology of ET, a genetics study, and a brain repository. Sensitivity was the proportion of ET cases with head tremor on examination who self-reported head tremor. Results: The sensitivity of the screening question for head tremor was lowest in the population-based study (31.6\%), and higher as well as somewhat similar across the remaining studies (46.7 to $62.2 \%$ ). Higher sensitivity was associated with tremor of longer duration, presence of voice tremor on examination, female gender, and lower education. The use of the screening question would have increased case ascertainment during the screening phase of these four studies by $1.9,4.1,10.2$, and $20.3 \%$.
Conclusions: A screening question for head tremor had lowto-moderate sensitivity in ET. The use of such a screening questionnaire, however, has the potential to increase case ascertainment by as much as $20 \%$ in some screening settings.

(c) 2014 S. Karger AG, Basel

\section{Introduction}

The initial step in much of epidemiological research is case ascertainment. In prevalence and incidence studies, complete ascertainment of cases is of prime importance. Nevertheless, case identification is often fraught with difficulties. In essential tremor (ET) research, for example, spiral drawing is a commonly employed method to screen populations. Hand-drawn spirals may easily be collected by email, facsimile, postal service and other methods, none of which require the investigator to travel, thereby saving time and resources when screening large numbers of people. However, some ET cases may not exhibit tremor in their hand-drawn spiral. Indeed, population-based studies estimate that $34.5 \%$ of ET cases have tremor that is either minimal or absent when drawing a spiral with their dominant

\section{KARGER}

E-Mail karger@karger.com www.karger.com/ned
(C) 2014 S. Karger AG, Basel

$0251-5350 / 14 / 0431-0065 \$ 39.50 / 0$
Elan Louis

Unit 198, Neurological Institute

710 West 168th Street

New York, NY 10032 (USA)

E-Mail EDL2@ columbia.edu 
arm [1]. Failure to detect one in three ET cases, when using this popular screening method, is highly problematic.

Head tremor is a common feature in ET, occurring in $10.9 \%$ to $18.0 \%$ of ET cases in population-based studies [2-4] and as many as $60.6 \%$ of cases in more selected samples such as brain repositories [5]. Therefore, screening for and identifying individuals with head tremor has the potential to boost case ascertainment in neuroepidemiological studies.

Thus, the question naturally arises, how valid are reports of head tremor among patients with ET? There are compelling reasons to expect that ET patients would not report head tremor even when it is present - ET patients are often completely unaware of their own head tremor. A proposed mechanism is that reduced sensitivity to head motion allows patients to achieve a sense of perceptual stability [6]. This type of perceptual cancelling occurs in patients with congenital nystagmus as well [7].

The primary aim of the current study was to assess the validity of a screening question for head tremor in ET. There are no published data on this topic, and the knowledge will guide future epidemiological investigations of this disorder. A second aim was to estimate the number of additional ET cases who would have been ascertained with the addition of a screening question for head tremor. In order to broadly assess this issue across a wide range of possible settings, these analyses utilized four distinct patient samples: a population-based study in northern Manhattan [8], a study of the environmental epidemiology of ET [9], a genetics study [10], and a brain repository [5]; in total, more than 800 ET patients were studied in these four parallel datasets.

\section{Methods}

Four ET case samples were used - cases all signed written informed consent as approved by our institutional ethics board.

\section{Population-Based Study in Northern Manhattan}

The Washington Heights-Inwood Genetic Study of Essential Tremor (WHIGET) was a family study of ET in the Washington Heights-Inwood community in northern Manhattan, New York. Enrollment was completed in 2000. There were 106 ET cases, including 59 probands with ET, 33 of their relatives with ET, and 14 affected relatives of control probands. The design of this population-based study has been described in detail $[8,11,12]$. All enrollees underwent a demographic and medical history and a videotaped tremor examination.

\section{Study of the Environmental Epidemiology of ET}

ET cases were enrolled in a study of the environmental epidemiology of ET, from 2000-2009 [9]. As described, cases were derived from two main sources: the Neurological Institute of New
York at Columbia University Medical Center (CUMC) and the membership of the International Essential Tremor Foundation (IETF). After enrollment, all 388 ET cases underwent a demographic and medical history and a videotaped tremor examination.

\section{Family Study of Essential Tremor}

ET cases (probands) and their first-and second-degree relatives were enrolled in the Family Study of Essential Tremor (FASET), a genetics study of ET at CUMC $[13,14]$. The study was advertised on two ET society websites. Based upon a telephone interview with the proband, relatives with ET were identified. The final sample included 207 individuals (52 probands and 155 relatives), including 160 ET, 28 borderline ET, and 19 normal [13]. After enrollment, subjects underwent a demographic and medical history and a videotaped tremor examination.

\section{Essential Tremor Centralized Brain Repository}

The Essential Tremor Centralized Brain Repository (ETCBR) at Columbia University is a centralized repository for the prospective collection and study of ET brains $[5,15]$. ET cases were recruited as future brain donors through the IETF and other sources. Once enrolled, they underwent a demographic and medical history and a videotaped tremor examination. Head tremor on videotaped examination was rated by a neurologist specializing in movement disorders (E.D.L.) as absent, mild and infrequent, mild or infrequent, moderate, or severe. These ET cases were highly selected because many were ascertained through a disease-specific organization and because they self-referred to the brain repository as future brain donors $[5,11]$. The large majority of these cases have seen physicians who have diagnosed ET [15].

\section{Medical History, Videotaped Tremor Examination and}

Diagnostic Confirmation

In all four studies, the medical history included questions on demographics, medications, and features of tremor, including the question, 'Does your head sometimes shake?' (in the Family Study of Essential Tremor) or 'Does your head often shake uncontrollably?' (in the remaining three studies). The videotaped tremor examination included assessments of postural and kinetic tremors in the arms, as well as head (neck), voice, and jaw tremors. This included one test for postural tremor and five for kinetic tremor (pouring, using spoon, drinking, finger-nose-finger, drawing spirals) performed with each arm (12 tests total). In each study, a neurologist specializing in movement disorders (E.D.L.) used a reliable [16] and valid [17] clinical rating scale, the WHIGET Tremor Rating Scale, to rate postural and kinetic tremor during each test: 0 (none), 1 (mild), 2 (moderate), 3 (severe). These ratings resulted in a total tremor score (range $=0-36$ ), which is an assessment of postural and kinetic tremor in the arms [9].

In each study, the diagnosis of ET was re-assessed (E.D.L.) based on the history and videotaped examination. All of the cases included in these analyses met published criteria for ET (moderate or greater amplitude kinetic tremor of the arms during at least three tasks or head tremor, in the absence of Parkinson's disease, dystonia or another neurological disorder) [12].

Statistical Analyses

Data on each of the four case samples were analyzed separately in SPSS (Version 21). Sensitivity was the proportion of ET cases with head tremor on examination who self-reported head 
Table 1. Demographic and clinical features of ET cases in four ET case samples

\begin{tabular}{|c|c|c|c|c|}
\hline Age in years & $69.8 \pm 18.4$ & $67.4 \pm 15.3$ & $60.0 \pm 18.0$ & $83.7 \pm 5.8$ \\
\hline Female gender & $63(59.4)$ & $201(51.8)$ & $82(51.3)$ & $108(61.0)$ \\
\hline Tremor duration in years & $15.4 \pm 18.8$ & $22.8 \pm 18.7$ & $32.8 \pm 19.3$ & $41.3 \pm 22.3$ \\
\hline Age of tremor onset in years & $56.7 \pm 25.6$ & $44.7 \pm 22.5$ & $27.0 \pm 17.8$ & $42.4 \pm 22.9$ \\
\hline Currently taking medication for tremor & $5(4.7)$ & $209(53.9)$ & $79(49.4)$ & $115(65.0)$ \\
\hline Family history of ET & $106(100)$ & $119(30.7)$ & $160(100)$ & $48(27.1)$ \\
\hline Voice tremor on examination & $16(15.1)$ & $118(30.4)$ & $26(16.3)$ & $71(40.1)$ \\
\hline Head tremor on examination & $19(17.9)$ & $143(36.9)$ & $60(37.5)$ & $92(52.0)$ \\
\hline head tremor on examination & 3 & 40 & 10 & 3 \\
\hline
\end{tabular}

All values are means \pm standard deviations or numbers (percentages).

tremor. Specificity was the proportion of ET cases without head tremor on examination who did not self-report head tremor. False positives were ET cases who self-reported head tremor but had no head tremor on examination. Estimates of the sensitivity included point estimates and $95 \%$ confidence intervals (CI). In a logistic regression model, we assessed the association between the dependent variable (reported head tremor in the setting of head tremor on examination) and head tremor severity category (independent variable, coded as absent, mild and infrequent, mild or infrequent, moderate, or severe). We also present data on a range of demographic clinical features that were of a priori interest because we thought that sensitivity could co-vary with these.

\section{Results}

Demographic and clinical features of ET cases are presented (table 1). As would be expected, the severity of arm tremor (total tremor score), the duration of tremor, and the prevalence of head tremor on examination was lowest in the population-based study in northern Manhattan and was highest in the Essential Tremor Centralized Brain Repository (table 1).

The sensitivity of the question on self-reported head tremor was lowest in the population-based study in northern Manhattan (31.6\%), and higher as well as some-

Validity of a Screening Question for Head

Tremor what similar across the remaining three studies $(46.7 \%$, $56.7 \%, 62.2 \%$, table 2 ).

In the Essential Tremor Centralized Brain Repository, the severity of head tremor on neurological examination was rated. With increasing head tremor severity, there was an increase in the sensitivity of the screening question (i.e., the proportion of ET cases with head tremor on examination who self-reported head tremor): 7 (28.0\%) of 25 with mild and infrequent head tremor, $11(39.3 \%)$ of 28 with mild or infrequent head tremor, $23(62.2 \%)$ of 37 with moderate head tremor, and $2(100 \%)$ of 2 with severe head tremor (odds ratio $=2.36,95 \%$ confidence interval $=$ $1.75-3.18, \mathrm{p}<0.001$ in a logistic regression model).

The specificity of self-reported head tremor was $84 / 87$ $(96.6 \%)$ in the population-based study in northern Manhattan, 203/245 (82.9\%) in the study of the environmental epidemiology of ET, 76/100 (76.0\%) in the Family Study of Essential Tremor, and 76/85 (89.4\%) in Essential Tremor Centralized Brain Repository; hence the proportion of false positives (ET cases who self-reported head tremor yet who did not have head tremor on examination) ranged from 3.4 to $24.0 \%$.

We explored a wide range of clinical features that could be predictive of increased sensitivity (that is, subgroups of patients who might be better historians) (table 2). We 
Table 2. Sensitivity of question on self-reported head tremor

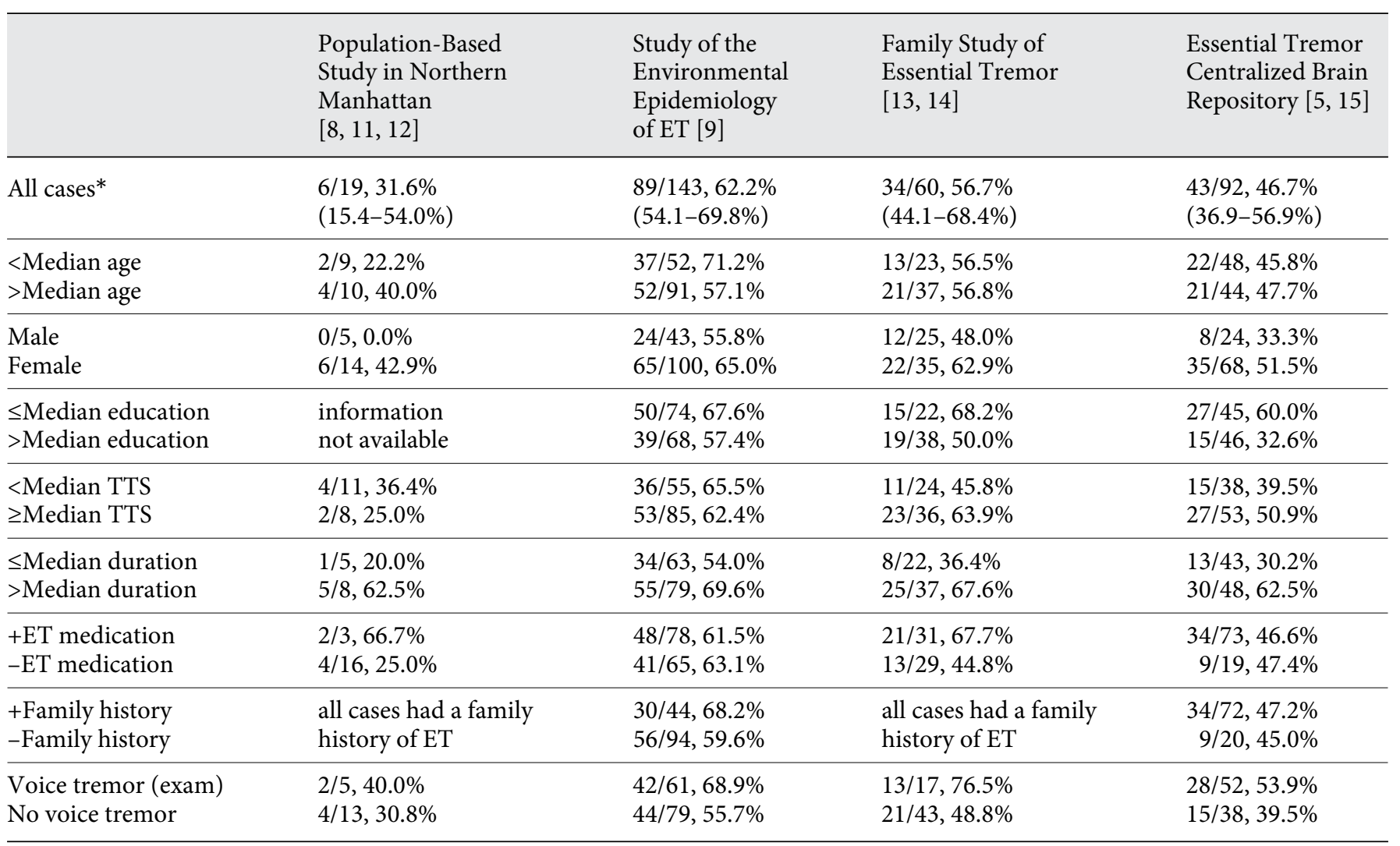

* Values are number with self-reported head tremor/number with head tremor on examination, prevalence per 100 ( $95 \%$ confidence interval). TTS $=$ Total tremor score. Some data points were missing for education, total tremor score, tremor duration, family history of $\mathrm{ET}$, and voice tremor on examination.

found that higher sensitivity was associated with tremors of longer duration, presence of voice tremor on examination, female gender, and lower education (table 2).

A major goal of screening for head tremor is to enhance the ascertainment of ET cases in epidemiological studies. With this in mind, in each study, we assessed the number of additional ET cases who would have been ascertained by adding a screening question for head tremor to an assessment of the hand-drawn spiral. During the screening stage of the population-based study in northern Manhattan, 74 ET cases would have been ascertained based on spiral drawings; three additional ET cases $(4.1 \%$ more) would have been ascertained by adding the screening question for head tremor (table 1). In the three other studies, this value was $20.3 \%$ (study of the environmental epidemiology of ET), 10.2\% (Family Study of Essential Tremor) and 1.9\% (Essential Tremor Centralized Brain Repository) (table 1).

\section{Discussion}

In the current study, we demonstrated that the sensitivity of a screening question for head tremor was very much dependent on the population being sampled, ranging from as low as $31.6 \%$ in a population-based study in northern Manhattan to as high as $62.2 \%$ in other studies. Hence, the sensitivity ranges from low to moderate. We also showed that the use of such a screening questionnaire has the potential to increase case ascertainment, in screening settings, by as much as $20 \%$ in some study settings.

The screening question had the lowest sensitivity in the population-based study in northern Manhattan. In general, the tremor observed in the population is milder than that in the other studies $[2,3]$. Indeed, only $4.7 \%$ of patients in our population-based study in northern Manhattan were taking medication for tremor. The tremor in 
our population-based study in northern Manhattan was also of relatively short duration. These factors may have contributed to a reduced awareness of head tremor in these cases.

We found a number of demographic and clinical features were associated with reports of greater validity: tremor of longer duration, presence of voice tremor on examination, female gender, and lower education. Having had tremor for longer duration might make patients more aware of or more in tune with their symptoms and signs. Additionally, the presence of other forms of cranial tremor (e.g., voice tremor) might increase the awareness of tremors in this general body region as well. Head tremor is more prevalent in women with ET than in men with ET [18], although this does not explain why women would be more likely to report tremor. One possible explanation is that published data indicate that women are better historians than men in a variety of medical settings ranging from self-reporting utilization of health services [19] to self-reporting stroke and acute myocardial infarction [20]. Moreover, head tremor is particularly noticeable and difficult to conceal. Given a greater susceptibility to body dissatisfaction [21], women might be more aware of and more likely to report head tremor than men.

The question we used to elicit tremor in three of the four studies was 'Does your head often shake uncontrollably?' In the Family Study of Essential Tremor, we encouraged the self-reporting of milder forms of head tremor ('Does your head sometimes shake?') Despite this difference, the sensitivity of self-reported tremor was similar in the Family Study of Essential Tremor and two of the three other studies.

The strengths of the study were that we utilized data on ET cases spanning a broad range of settings, from a population-based study to a highly-selected sample of brain donors, with our total sample size being in excess of
800. All cases underwent a detailed videotaped tremor examination, which was carefully assessed by a senior neurologist specializing in movement disorders. In three of the studies, the screening question was identical; in a fourth, we used a less restrictive question, allowing us to assess validity in this situation as well.

In summary, a screening question for head tremor had low-to-moderate sensitivity in ET. The use of such a screening questionnaire, however, has the potential to increase case ascertainment by as much as $20 \%$ in some screening settings.

\section{Acknowledgements}

This work was supported by NIH grants R01 NS039422, R01 NS042859, R01 NS073872 and UL1 TR000040. These research aims otherwise received no specific grant from any funding agency in the public, commercial or not-for-profit sectors.

\section{Disclosure Statement}

The authors declare that there are no conflicts of interest and no competing financial interests.

\section{Contributions}

Elan D. Louis MD MSc: study conception and design, analysis and interpretation of data, study supervision, initial draft and critical revision of manuscript for important intellectual content.

Monika Michalec MPH: study design, critical revision of manuscript for important intellectual content.

\section{Statistical Analyses}

The statistical analyses were conducted by Dr. Louis.

\section{References}

1 Louis ED, Ford B, Wendt KJ, Lee H, Andrews $\mathrm{H}$ : A comparison of different bedside tests for essential tremor. Mov Disord 1999;14:462467.

$\checkmark 2$ Dogu O, Louis ED, Sevim S, Kaleagasi H, Aral M: Clinical characteristics of essential tremor in Mersin, Turkey - a populationbased door-to-door study. J Neurol 2005; 252:570-574

-3 Louis ED, Ford B, Wendt KJ, Cameron G: Clinical characteristics of essential tremor: data from a community-based study. Mov Disord 1998;13:803-808.

Validity of a Screening Question for Head Tremor
4 Benito-León J, Bermejo-Pareja F, Morales JM, Vega S, Molina JA: Prevalence of essential tremor in three elderly populations of central Spain. Mov Disord 2003;18:389-394.

5 Louis ED, Borden S, Moskowitz CB: Essential tremor centralized brain repository: diagnostic validity and clinical characteristics of a highly selected group of essential tremor cases. Mov Disord 2005;20:1361-1365.

6 6 Louis ED, Pellegrino KM, Rios E: Unawareness of head tremor in essential tremor: a study of three samples of essential tremor patients. Mov Disord 2008;23:2423-2424.
7 Lee AG, Brazis PW: Localizing forms of nystagmus: symptoms, diagnosis, and treatment. Curr Neurol Neurosci Rep 2006;6: 414-420.

$>8$ Louis ED, Ford B, Frucht S: Factors associated with increased risk of head tremor in essential tremor: a community-based study in northern Manhattan. Mov Disord 2003;18:432436

$>9$ Louis ED, Zheng W, Applegate L, Shi L, Factor-Litvak P: Blood harmane concentrations and dietary protein consumption in essential tremor. Neurology 2005;65:391-396. 
10 Louis ED, Hernandez N, Ionita-Laza I, Ottman R, Clark LN: Does rate of progression run in essential tremor families? Slower vs. faster progressors. Parkinsonism Relat Disord 2013;19:363-366.

11 Louis ED, Rios E, Applegate LM, Hernandez NC, Andrews HF: Jaw tremor: prevalence and clinical correlates in three essential tremor case samples. Mov Disord 2006;21:1872-1878.

12 Louis ED, Ottman R, Ford B, Pullman S, Martinez M, Fahn S, Hauser WA: The Washington Heights-Inwood Genetic Study of Essential Tremor: methodologic issues in essentialtremor research. Neuroepidemiology 1997; 16:124-133.

13 Louis ED, Ottman R, Clark LN: Clinical classification of borderline cases in the family study of essential tremor: an analysis of phenotypic features. Tremor Other Hyperkinet Mov (N Y) 2014;4:220.
14 Louis ED, Hernandez N, Rabinowitz D, Ottman R, Clark LN: Predicting age of onset in familial essential tremor: how much does age of onset run in families? Neuroepidemiology 2013;40:269-273.

15 Babij R, Lee M, Cortés E, Vonsattel JP, Faust PL, Louis ED: Purkinje cell axonal anatomy: quantifying morphometric changes in essential tremor versus control brains. Brain 2013; 136(Pt 10):3051-3061.

16 Louis ED, Ford B, Bismuth B: Reliability between two observers using a protocol for diagnosing essential tremor. Mov Disord 1998; 13:287-293.

17 Louis ED, Wendt KJ, Albert SM, Pullman SL, $\mathrm{Yu} \mathrm{Q}$, Andrews H: Validity of a performancebased test of function in essential tremor. Arch Neurol 1999;56:841-846.

18 Hardesty DE, Maraganore DM, Matsumoto JY, Louis ED: Increased risk of head tremor in women with essential tremor: longitudinal data from the Rochester Epidemiology Project. Mov Disord 2004;19:529-533.
9 Kim H, Lee K, Chang S, Kang G, Tak Y, Lee M, Kim V, Lee J, Jeong H: Factors affecting the validity of self-reported data on health services from the community health survey in Korea. Yonsei Med J 2013;54:1040-1048.

20 Machón M, Arriola L, Larrañaga N, Amiano P, Moreno-Iribas C, Agudo A, Ardanaz E, Barricarte A, Buckland G, Chirlaque MD, Gavrila D, Huerta JM, Martínez C, Molina E, Navarro C, Quiros JR, Rodríguez L, Sanchez MJ, González CA, Dorronsoro M: Validity of self-reported prevalent cases of stroke and acute myocardial infarction in the Spanish cohort of the EPIC study. J Epidemiol Community Health 2013;67:71-75.

21 Quick VM, Byrd-Bredbenner C: Disordered eating, socio-cultural media influencers, body image, and psychological factors among a racially/ethnically diverse population of college women. Eat Behav 2014;15:37-41. 